\title{
Erratum: Spin Transport in Nondegenerate Si with a Spin MOSFET Structure at Room Temperature [Phys. Rev. Appl. 2, 034005 (2014)]
}

\begin{abstract}
Tomoyuki Sasaki, Yuichiro Ando, Makoto Kameno, Takayuki Tahara, Hayato Koike, Tohru Oikawa, Toshio Suzuki, and Masashi Shiraishi
\end{abstract}

(Q) (Received 4 February 2018; published 6 March 2018)

DOI: 10.1103/PhysRevApplied.9.039901

The authors identified an insufficient description in the caption of Fig. 3(a) of the main text about subtracting a background signal to obtain the Hanle spin signals [Fig. 3(a)]. The detailed procedure is as follows: The background signal was defined as a signal that was independent of magnetization alignments, i.e., parallel and antiparallel. The background signal $V_{\mathrm{BG}}(B)$ was calculated to be $\left[V_{P}(B)+V_{\mathrm{AP}}(B)\right] / 2$, where $V_{P}(B)$ and $V_{\mathrm{AP}}(B)$ are Hanle spin signals under parallel and antiparallel magnetization configurations, under the assumption that the background signal is unchanged. The authors

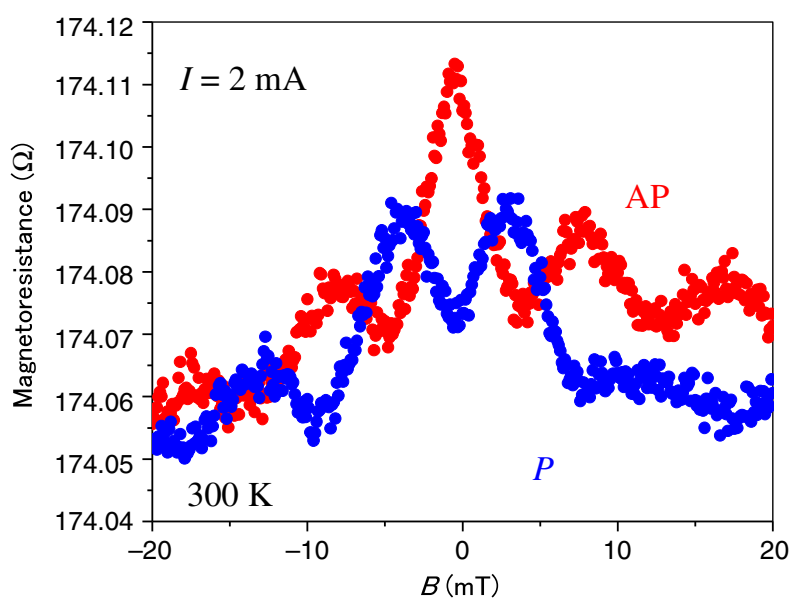

FIG. 1. Raw Hanle signals measured at $300 \mathrm{~K}$. The signal reversal of the signals under the parallel and antiparallel magnetization configuration is apparently seen.
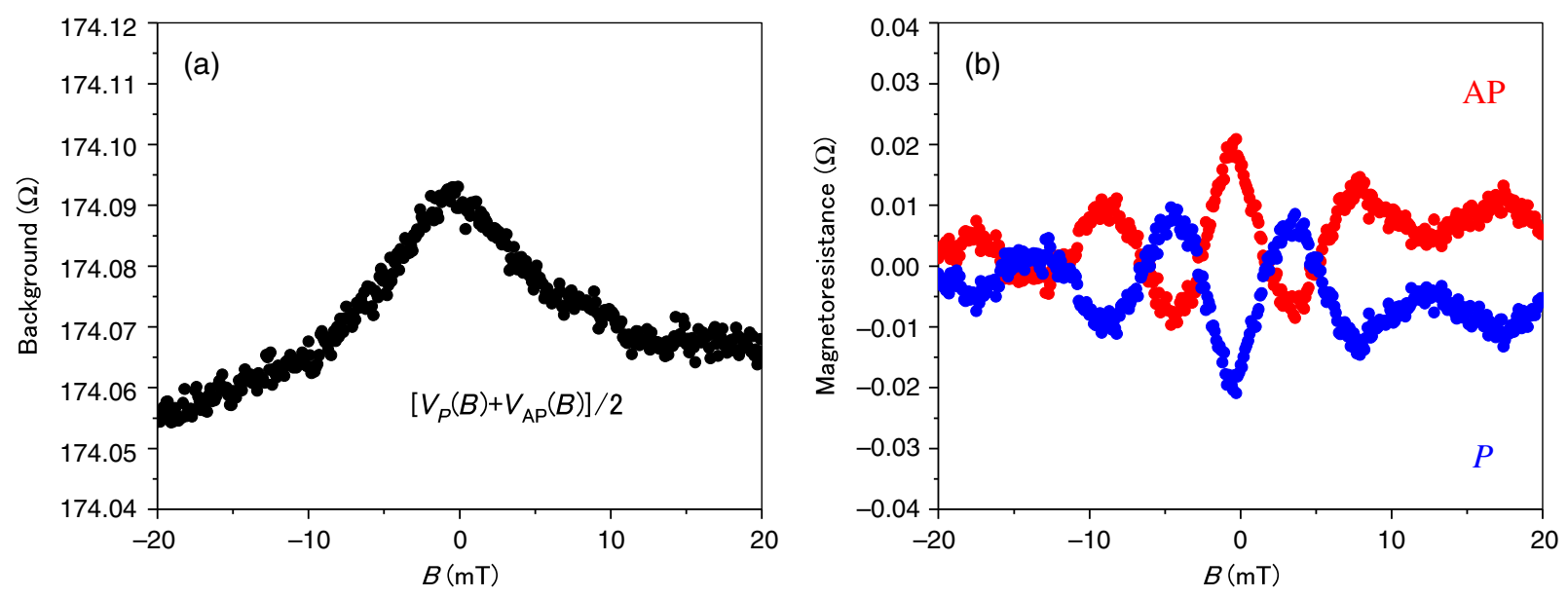

FIG. 2. (a) The extracted background signal by using the procedure in the text. (b) The Hanle signals after subtracting the background signal shown in (a) of this figure. 
subtracted this background signal from raw Hanle spin signals under the parallel and the antiparallel configurations. Consequently, the net Hanle signal under the parallel configuration is calculated to be $\left[V_{P}(B)-V_{\mathrm{BG}}(B)\right]=$ $\left[V_{P}(B)-V_{\mathrm{AP}}(B)\right] / 2$, and the net spin signal under the antiparallel configuration is calculated to be $\left[V_{\mathrm{AP}}(B)-V_{\mathrm{BG}}(B)\right]=$ $\left[V_{\mathrm{AP}}(B)-V_{P}(B)\right] / 2$. As can be seen, the net Hanle spin signals under the parallel and the antiparallel configurations exhibited the opposite polarity and the same signal amplitude, which means either Hanle signal has less physical meaning. Meanwhile, it is noted that the Hanle spin signal after the subtraction of the background signal showed the zero crossing (at ca. $\pm 3,6, \ldots \mathrm{mT})$, which indicates the signals were attributed to the Hanle effect. Although the procedure for subtracting the background signal was already described in the caption of Fig. 3, the authors identified that this insufficient description of the procedure might induce unnecessary confusion for readers.

To avoid further unnecessary confusion, the authors also show raw Hanle signals in the new Fig. 1 presented in this Erratum, where signal inversion under the parallel and antiparallel configurations is obviously seen. The new Figs. 2(a) and 2(b) presented here show the extracted background signal by using the above-mentioned procedure and the Hanle signals after subtracting this background signal, respectively. As can be seen, the background signal cannot be described by a specific analytic function, which is the reason why the authors adopted this procedure to subtract a background signal, otherwise, the estimated spin-transport parameters, such as spin-diffusion length, etc., include sizable errors. Linear background signals are still superposed to the Hanle signals shown in Fig. 2(b). The origin of these backgrounds is most likely due to time-dependent drifting of the offset voltages, which sometimes takes place in Hanle measurements. In fact, the slopes of them are opposite under the parallel and the antiparallel configurations. After subtracting the linear background from each Hanle signal, the Hanle signals shown in Fig. 3(a) in the main text were obtained. 